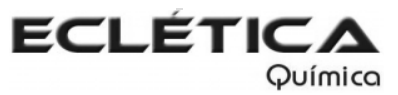

www.scielo.br/eq

Volume 31, número 1, 2006

\title{
Síntese e caracterização de complexos de platina (II) contendo iodeto e derivados do furano como ligantes
}

\author{
W. Guerral ${ }^{l}$ H. Silval, M. V. de Almeidal e A. P. S. Fontes ${ }^{1 *}$ \\ ${ }^{\prime}$ Departamento de Química, ICE, Universidade Federal de Juiz de Fora, Campus Martelos, \\ 36036-330 Juiz de Fora - MG \\ *e-mail:ana.fontes@ufjf.edu.br
}

Resumo: Alguns compostos de platina (II), como por exemplo, cisplatina, carboplatina e oxaloplatina, têm sido utilizados como agentes quimioterápicos no tratamento de vários tipos de câncer. Contudo, na tentativa de reduzir a toxicidade e ampliar o espectro de atividade da cisplatina e de seus análogos, milhares de complexos têm sido preparados variando a natureza dos ligantes. Este trabalho descreve a síntese e caracterização de novos complexos de platina, tendo iodeto e derivados do furano como ligantes. Os complexos foram caracterizados por RMN, IV e análise elementar.

Palavras-chave: complexos de platina; oximas; semicarbazonas; furano.

\section{Introdução}

Cis- $\left\{\mathrm{Pt}\left(\mathrm{NH}_{3}\right)_{2} \mathrm{Cl}_{2}\right\}$, cisplatina, é eficiente no tratamento de diversos tipos de câncer como o de pulmão, cabeça, estômago e em especial o de ovário e testículo, sendo que para este último tem-se conseguido aproximadamente $90 \%$ de cura [1-4]. Sobretudo a cisplatina é utilizada em associações com outras drogas em vários esquemas terapêuticos $[5,6]$.

Contudo, os efeitos tóxicos associados a este composto, o limitado espectro de atividade e o aparecimento de resistência celular levaram ao desenvolvimento de uma série de análogos, dentre eles a carboplatina e oxaloplatina, que estão disponíveis comercialmente. Em relação à cisplatina, a grande vantagem destes análogos diz respeito à diminuição da nefrotoxicidade, permitindo o uso de doses maiores. Entretanto, quanto ao espectro de atividade, eles são um pouco menos eficientes do que a cisplatina [5]. Assim vários outros complexos de platina estão sendo desenvolvidos e novos tipos estruturais têm emergido dos laboratórios de pesquisa. A alteração do grupo abandonador tem revelado resultados interessantes, inclusive na redução da toxicidade $[2,7]$.

Neste trabalho utilizou-se os ligantes 2furanocarboxaldeído oxima, ligante a, e 2-furanocarboxaldeído semicarbazona, ligante $\mathbf{b}$, figura 1. Oximas e seus complexos apresentam atividade antiviral e exercem atividade citotóxica em linhagens celulares humanas e em tumores sólidos [8]. Semicarbazonas e seus complexos metálicos apresentam diversas atividades biológicas, como exemplo antitumoral e antiviral [9].

Este trabalho reporta parte da estratégia de nosso grupo de pesquisa na obtenção de novos complexos de platina variando a natureza dos ligantes. Recentemente, publicamos um trabalho envolvendo a preparação de complexos de platina (II) contendo derivados do furano e cloreto como ligantes [6]. 


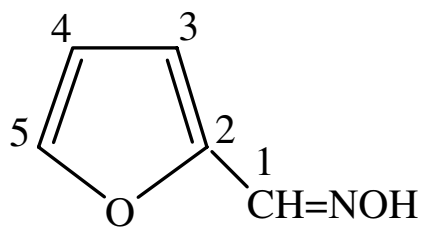

ligante a

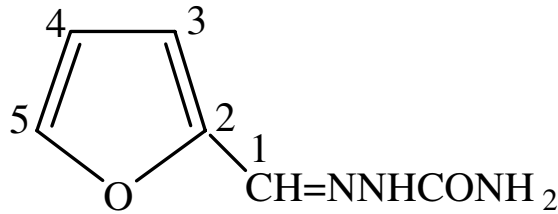

ligante $b$

Figura 1.

\section{Material e Métodos}

Sintese dos ligantes

2-furanocarboxaldeído oxima (a)

Uma mistura de $0,840 \mathrm{~g}$ de bicarbonato de sódio com 0,695 $\mathrm{g}$ de cloridrato de hidroxilamina em metanol e água, foi acrescentada a uma solução de $0,82 \mathrm{~mL}$ ( $10 \mathrm{mmols} ; 0,96 \mathrm{~g})$ de furfural em $20 \mathrm{~mL}$ de metanol e a mistura resultante permaneceu sob agitação durante 24 horas. Após este tempo, evaporou-se o solvente e com o sólido obtido, redissolvido em diclorometano, fez-se uma coluna (eluente: acetato/ hexano 2:8). O composto puro foi obtido nas primeiras frações como um sólido cristalino branco, com rendimento de $58 \%(0,64 \mathrm{~g})$.

IV. $v \mathrm{KBr}\left(\mathrm{cm}^{-1}\right): 3286(v \mathrm{OH}), 3080\left(v_{\mathrm{s}}\right.$ $\left.\mathrm{CH}_{\text {fur. }}\right), 3041\left(v_{\text {as }} \mathrm{CH}_{\text {fur. }}\right), 2860\left(v \mathrm{CH}_{\text {alif. }}\right), 1646$ $(v \mathrm{C}=\mathrm{N}), 1478(v \mathrm{NOH}), 1144(v \mathrm{NO}), 1017(v$ $\mathrm{C}-\mathrm{O}-\mathrm{C}$ ).

RMN de ${ }^{1} \mathrm{H}\left(200 \mathrm{MHz} ; \mathrm{CD}_{3} \mathrm{OD}\right) \delta 7,39 ;$ 4,88 (2s, $\mathrm{HCl}$ e $\mathrm{H}$ do grupo $\mathrm{OH}$ ); 7,52; 6,53 (2dd, HC5 e HC4, $\mathrm{J}_{1-2}=0,62 \mathrm{~Hz}, \mathrm{~J}_{1-3}=1,76 \mathrm{~Hz}$ ); 7,26 (d, HC3, J= 3,4 Hz).

$\mathrm{RMN}$ de ${ }^{13} \mathrm{C}\left(50 \mathrm{MHz} ; \mathrm{CD}_{3} \mathrm{OD}\right) \delta 112$, 118, 144 e 137 (C4, C3, C5 e C1); 147 (C2).

\section{2-furanocarboxaldeído semicarbazona (b)}

Uma solução com $1,0 \mathrm{~g}$ de cloridrato de semicarbazida e $1,5 \mathrm{~g}$ de acetato de sódio dissolvidos em água foi adicionada sob agitação a
$0,5 \mathrm{~mL}$ de furfural $(6 \mathrm{mmols}, 0,59 \mathrm{~g})$ a $0{ }^{0} \mathrm{C}$. A reação ocorreu instantaneamente, produzindo um sólido amarelado. O sólido foi filtrado e lavado com éter etílico e depois de seco foi pesado, obtendo-se um rendimento de $82 \%$ (0,77 g).

IV. $v \mathrm{KBr}\left(\mathrm{cm}^{-1}\right): 3459\left(v_{\mathrm{s}} \mathrm{NH}_{2}\right), 3279\left(v_{\mathrm{as}}\right.$ $\left.\mathrm{NH}_{2}\right), 3180\left(v_{\mathrm{s}} \mathrm{NH}\right), 3160\left(v_{\mathrm{as}} \mathrm{NH}\right), 3081\left(v_{\mathrm{s}} \mathrm{CH}\right.$ fur. $), 3008\left(v_{\text {as }} \mathrm{CH}_{\text {fur. }}\right), 2852\left(v \mathrm{CH}_{\text {alif. }}\right), 1696(v$ $\mathrm{C}=\mathrm{O}), 1658(v \mathrm{C}=\mathrm{N}), 1007$ ( $v \mathrm{C}-\mathrm{O}-\mathrm{C})$.

RMN de ${ }^{1} \mathrm{H}\left(200 \mathrm{MHz}\right.$; Acetona- $\left.d_{6}\right) \delta$ 10,23; 7,74 (2s, HC1 e H do grupo $\mathrm{NH}) ; 6,31(\mathrm{H}$ do grupo $\mathrm{NH}_{2}$ ); 7,73 (HC5); 6,70; 6,60 (d e dd, $\mathrm{HC} 3$ e $\mathrm{HC} 4$ ).

\section{Síntese dos Complexos}

Em uma solução de $0,5 \mathrm{mmol}(0,208 \mathrm{~g}) \mathrm{de}$ $\mathrm{K}_{2} \mathrm{PtCl}_{4}$ dissolvidos em $5 \mathrm{~mL}$ de água, foi adicionado 2 mmols $(0,332 \mathrm{~g})$ de KI dissolvidos em 5 $\mathrm{mL}$ de água. A solução foi mantida sob agitação durante 25 minutos. Após este tempo, foi adicionado in situ $1 \mathrm{mmol}(0,111 \mathrm{~g})$ de 2-furanocarboxaldeído oxima dissolvidos com o auxílio do ultra-som em $20 \mathrm{~mL}$ de água, para complexo I, ou $1 \mathrm{mmol}(0,153 \mathrm{~g})$ de 2-furanocarboxaldeído semicarbazona em $13 \mathrm{~mL}$ de água, para complexo II. A reação foi mantida sob agitação durante 24 horas. Após este tempo, filtrou-se o precipitado, que foi lavado com metanol e água. Obteve-se $0,236 \mathrm{~g}$ do complexo I (rendimento de $70 \%$ ), e 0,200 g do complexo II (rendimento de 66\%).

I- I.V. $v \mathrm{KBr}\left(\mathrm{cm}^{-1}\right): 3210(v \mathrm{OH}), 3018(v$ $\left.\mathrm{CH}_{\text {fur. }}\right), 2860\left(v \mathrm{CH}_{\text {alif. }}\right), 1615(v \mathrm{C}=\mathrm{N}), 1469(v$ $\mathrm{NOH}), 1144$ ( $v$ NO), 1027 (C-O-C), 571 ( $v$ Pt-N). Análise elementar calculada para $\mathrm{C}_{10} \mathrm{H}_{10} \mathrm{O}_{4} \mathrm{~N}_{2} \mathrm{PtI}_{2}$ : C 17,83; H 1,49; N 4,16; Pt 29,06. Encontrada: C 17,90; H 1,52; N 4,11; Pt 28,58.

RMN de ${ }^{195} \mathrm{Pt}$ (86 MHZ; DMF- $d_{7}$ ): $v$ 3393.23.

II- I.V. $v$ KBr $\left(\mathrm{cm}^{-1}\right): 3458\left(v_{\mathrm{s}} \mathrm{NH}_{2}\right), 3279$ $\left(v_{\text {as }} \mathrm{NH}_{2}\right), 3180\left(v_{\mathrm{s}} \mathrm{NH}\right), 3158\left(v_{\mathrm{as}} \mathrm{NH}\right), 3108\left(v_{\mathrm{s}}\right.$ $\left.\mathrm{CH}_{\text {fur }}\right), 3009\left(v_{\text {as }} \mathrm{CH}_{\text {fur. }}\right), 2852\left(v \mathrm{CH}_{\text {alif. }}\right), 1686$ $(v \mathrm{C}=\mathrm{O}), 1650(v \mathrm{C}=\mathrm{N}), 1011$ ( $v \mathrm{C}-\mathrm{O}-\mathrm{C}), 527$ ( $v$ Pt-N). Análise elementar calculada para $\mathrm{C}_{6} \mathrm{H}_{7} \mathrm{O}_{2} \mathrm{~N}_{3} \mathrm{PtI}_{2}$ : C 11,96; H 1,16; N 6,98; Pt 32,39. Encontrada: C 11,99; H 1,12; N 7,02; Pt 31,44.

RMN de ${ }^{195} \mathrm{Pt}\left(86 \mathrm{MHZ}\right.$; DMF- $\left.d_{7}\right): v-$ 2931,07 .

Todos reagentes e solventes utilizados neste trabalho tinham grau de pureza P.A. e 
foram adquiridos da Aldrich e Merck, tendo sido também usados sem prévia purificação.

Os espectros na região do IV foram medidos no DQ da UFJF num espectrofotômetro Bomem FTIR MB-102, na região de 4000 a 300 $\mathrm{cm}^{-1}$, utilizando como suporte pastilhas de $\mathrm{KBr}$. Resolução: $4 \mathrm{~cm}^{-1}$.

Os resultados de análise elementar dos compostos foram obtidos na Central Analítica do Instituto de Química da USP. As análises de Pt por absorção atômica foram feitas num espectrofotômetro Hitachi 8200, no DQ da UFMG.

Os espectros de $\mathrm{RMN}$ de ${ }^{1} \mathrm{H}$, de ${ }^{13} \mathrm{C}$ e de ${ }^{195} \mathrm{Pt}$ foram feitos no DQ da UFMG utilizando um espectrômetro Brucker Avance DRX 400. Os deslocamentos químicos foram expressos a partir do padrão interno TMS (RMN de $\left.{ }^{1} \mathrm{H}\right)$ e de uma solução de $\mathrm{K}_{2} \mathrm{PtCl}_{4}$ em $\mathrm{D}_{2} \mathrm{O}$ ( $\mathrm{RMN}$ de ${ }^{195} \mathrm{Pt}$ ).

\section{Resultados e discussão}

Os ligantes utilizados neste trabalho, 2furanocarboxaldeído oxima, a, e 2-furanocarboxaldeído semicarbazona, b, foram sintetizados de acordo com o esquema 1. Para a obtenção do ligante a, fez-se a reação de 2-furanocarboxaldeído com cloridrato de hidroxilamina em metanol . Este ligante foi purificado por coluna cromatográfica utilizando como eluente diclorometano e metanol. A síntese e a caracterização do ligante b já foi anteriormente descrita por nós [6]. Os ligan- tes foram caracterizados por análise elementar, IR, RMN de ${ }^{1} \mathrm{H}$ e $\mathrm{RMN}$ de ${ }^{13} \mathrm{C}$.

$\mathrm{O}$ espectro no infravermelho do ligante a apresentou bandas de absorção em 3286 e 3168 $\mathrm{cm}^{-1}$, correspondentes aos $v_{\mathrm{s}}$ do grupo $\mathrm{OH}$. Uma banda de absorção em $1646 \mathrm{~cm}^{-1}$ corresponde ao estiramento do grupo $\mathrm{C}=\mathrm{N}$.

A análise do espectro de $\mathrm{RMN}$ de ${ }^{1} \mathrm{H}$ nos revelou a presença de um simpleto em $v$ 7,39 referente ao hidrogênio 1 , um dupleto em $v 7,26$ $(\mathrm{J}=3,40 \mathrm{~Hz})$ e um dupleto duplo a $v 6,53\left(\mathrm{~J}_{1-2}=\right.$ $0,62 \mathrm{~Hz}$ e $\mathrm{J}_{13}=1,76 \mathrm{~Hz}$ ) correspondentes aos hidrogênios 3 e 4 , e outro dupleto duplo em $v$ $7,52\left(\mathrm{~J}_{1-2}=0,62 \mathrm{~Hz}\right.$ e $\left.\mathrm{J}_{1-3}=1,76 \mathrm{~Hz}\right)$ referente ao hidrogênio 5 .

$\mathrm{O}$ espectro de $\mathrm{RMN}$ de ${ }^{13} \mathrm{C}$ revela a presença de sinais em v112, 118, 137, 144 e 147 referentes aos carbonos do anel furânico C4, C3, $\mathrm{C} 1, \mathrm{C} 5$ e $\mathrm{C} 2$ respectivamente.

Para a síntese dos complexos partiu-se do precursor $\mathrm{K}_{2}\left[\mathrm{PtI}_{4}\right]$ que foi preparado a partir da reação de $\mathrm{K}_{2}\left[\mathrm{PtCl}_{4}\right]$ com iodeto de potássio. Os complexos obtidos, figura 2 , são sólidos de cor amarelo, sendo solúveis em dimetilssulfóxido (DMSO) e dimetilformamida (DMF).

Além da análise elementar e absorção atômica, os complexos foram caracterizados por $\mathrm{IV}$, por RMN de ${ }^{1} \mathrm{H}$ e $\mathrm{RMN}$ de ${ }^{13} \mathrm{C}$ e por $\mathrm{RMN}$ de 195Pt. Os resultados de análise elementar e absorção corroboram com as formulações propostas.

No espectro no infravermelho do complexo I pode-se observar que a banda referente ao

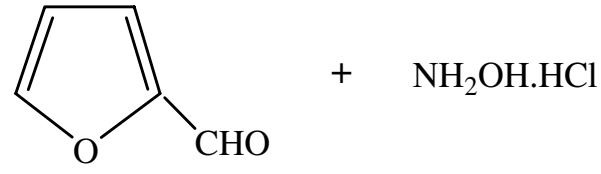

2-furanocarboxaldeído

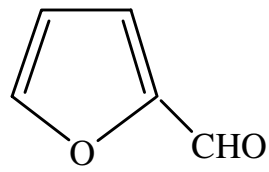

2-furanocarboxaldeído
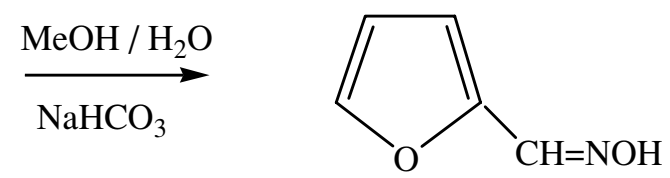

ligante a

Esquema 1. 

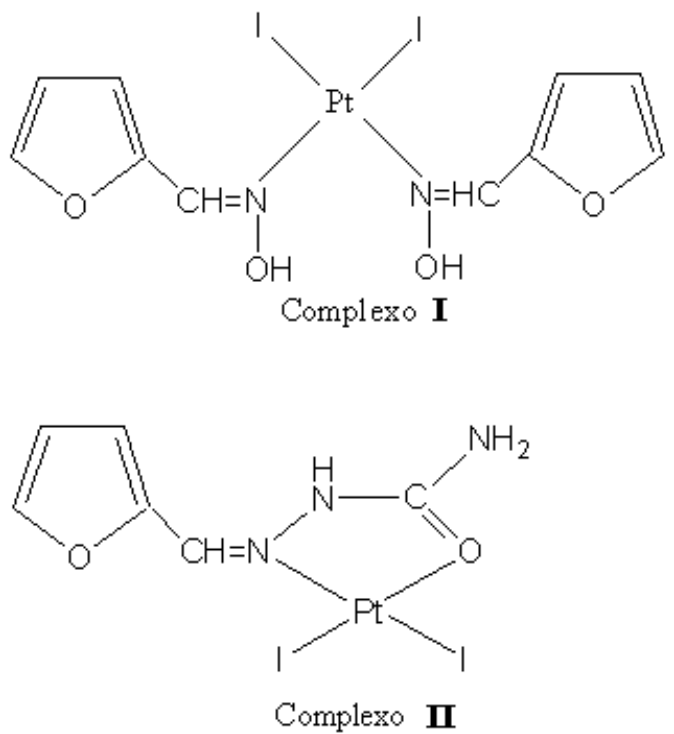

Figura 2.

$v_{\mathrm{C}=\mathrm{N}}$ do complexo encontra-se deslocada e com menor intensidade quando comparada com o espectro do ligante livre. De1646 $\mathrm{cm}^{-1}$ ela se desloca para $1615 \mathrm{~cm}^{-1}$ indicando que o metal se coordena pelo nitrogênio.

No espectro no infravermelho do complexo II pode-se observar que as bandas referentes aos $v_{\mathrm{C}=\mathrm{N}}$ e $v_{\mathrm{C}=\mathrm{O}}$ do complexo encontram-se deslocadas quando comparadas com o espectro do ligante livre. De 1696 e $1658 \mathrm{~cm}^{-1}$ estas bandas se deslocam para 1686 e $1650 \mathrm{~cm}^{-1}$, respectivamente, indicando que o metal se coordena pelo oxigênio do grupo $\mathrm{C}=\mathrm{O}$ e pelo nitrogênio do grupo $\mathrm{C}=\mathrm{N}$. Outra possibilidade seria a coordenação do metal ao nitrogênio do grupo $\mathrm{NH}_{2}$. Entretanto, o espectro de infravermelho do complexo, quando comparado ao do ligante livre, mostra que não há deslocamento das bandas de absorção referentes ao estiramento deste grupo, indicando que o mesmo não se encontra coordenado ao metal.

Os complexos aqui estudados apresentam novas bandas de absorção em $571 \mathrm{~cm}^{-1}$ e $527 \mathrm{~cm}^{-}$ ${ }^{1}$ que podem ser atribuídas aos $v_{\mathrm{Pt}-\mathrm{N}}$ para complexo I e II, respectivamente. Para o complexo II ainda observa-se uma nova banda em $408 \mathrm{~cm}^{-1}$ que pode ser atribuída ao $v_{\text {Pt-O }}[10]$.
Os espectros de $\mathrm{RMN}$ de ${ }^{1} \mathrm{H}$ e $\mathrm{RMN}$ de ${ }^{13} \mathrm{C}$ dos complexos foram obtidos. Entretanto, estes espectros não forneceram informações adicionais úteis para a caracterização do mesmo, estando, porém, coerente com a estrutura proposta.

Os complexos de platina foram caracterizados por RMN de ${ }^{195} \mathrm{Pt}$ em DMF para evitar a solvólise em DMSO, fenômeno bastante comum e bem documentado na literatura [11, 12].

$\mathrm{O}$ espectro de RMN de ${ }^{195} \mathrm{Pt}$ para o complexo I mostra um sinal em $v-3393$. Este valor de deslocamento químico está condizente com complexos similares encontrados na literatura, nos quais a platina tem na sua esfera de coordenação dois iodetos e dois átomos de nitrogênio $[13,14]$. Em comparação com o complexo I, o valor de deslocamento químico do sinal de ressonância encontrado em $\mathrm{RMN}$ de ${ }^{195} \mathrm{Pt}$ para o complexo II $(v-2931)$ encontra-se deslocado para campo baixo, o que está coerente com a estrutura proposta já que, no segundo complexo, a platina apresenta em sua esfera de coordenação um átomo de oxigênio, além de dois iodetos e de um átomo de nitrogênio.

\section{Conclusões}

Este trabalho descreveu a síntese de dois novos complexos de platina contendo iodeto e derivados do furano como ligantes. As reações para obtenção dos compostos foram feitas sob condições brandas e com bons rendimentos. As estruturas dos complexos obtidos podem ser modificadas tendo em vista que o iodeto é um bom grupo abandonador, podendo ser substituído por outro ligante.

Os complexos foram caracterizados pelas técnicas usuais de análise.

\section{Agradecimentos}

Os autores agradecem ao CNPQ, à CAPES e FAPEMIG pelo suporte financeiro.

Recebido em: 02/01/2006

Aceito em: 06/03/2006 
W. Guerra, H. Silva, M. V. de Almeida and A. P. S. Fontes. Synthesis and characterization of platinum (II) complexes containing iodide and furan derivatives as ligands.

\begin{abstract}
Platinum (II) compounds, for example, cisplatin, carboplatin and oxaloplatin, have been used as chemotherapeutic agents for the treatment of various types of cancer. However, in an attempt to reduce toxicity and widen the spectrum of activity of cisplatin and its analogous, thousands of platinum complexes have been prepared by varying the nature of the leaving groups and the carrier ligands. This work reports the synthesis and characterization of novel structural types of the platinum compounds, having iodide and furan derivatives as ligands. The compounds were characterized using NMR, IR and elemental analysis.
\end{abstract}

Keywords: platinum complexes; oximes; semicarbazones; furan.

\section{Referências}

[1] C. X. Zhang, S. J. Lippard, Current Opinion in Chem. Biol.. 7 (2003) 481.

[2] F. Huq, H. Daghriri, P. Beale, J. Inorg. Biochem. 98 (2004) 1261.

[3] M. Hidalgo, S. G. Eckhardt, J. Natl. Câncer Inst. 93 (2001) 178.

[4] V. Brabec, J. Kasparkova, Drug resistance Updat. 8 (2005) 131.

[5] B. Desoize, C. Madoulet, Crit. Rev. in Onc. /Hemat. 48 (2002) 317.

[6] W. Guerra, A. P. S. Fontes, M. V. de Almeida, H. Silva, Quím. Nova. 28 (2005) 809.
[7] A. P. S. Fontes, S. G. de Almeida, L. Nader, Quím. Nova. 20 (1997) 398.

[8] G. Ibrahim, J. Inog. Biochem. 81 (2000) 24.

[9] H. Beraldo, N. F. Nacif, W. Douglas, Spectrochim. Acta. 57 (2001) 1847.

[10] K. Nakamoto, K; Infrared and Raman Spectra of Inorganic and Coordination Compounds, Wiley, New York, 3rd ed., 1976.

[11] S. Kerrison, P. J. Sadler, Inorg. Chim acta. 104 (1985) 197.

[12] F. P. Fanizzi, F. L. Maresca, G. Natile, Inorg. Chem. 29 (1990) 29.

[13] F. D. Rochon, L. M. Gruia, Inorg. Chim. Acta. 306 (2000) 193.

[14] C.Tessier, F. D. Rochon, Inorg. Chim. Acta. 295 (1999) 25. 